\title{
Results from the South Pole Infra-Red EXplorer Telescope
}

\author{
J. M. Rathborne \\ Institute for Astrophysical Research, 725 Commonwealth Avenue, \\ Boston University, Boston, MA 02215, USA \\ School of Physics, University of New South Wales, Sydney, NSW 2052, \\ Australia
}

\section{G. Burton}

School of Physics, University of New South Wales, Sydney, NSW 2052, Australia

\begin{abstract}
The SPIREX telescope, located at the Amundsen-Scott South Pole Station, was a prototype system developed to exploit the excellent conditions for IR observing at the South Pole. Observations over two winter seasons achieved remarkably deep, high-resolution, wide-field images in the 3-5 $\mu \mathrm{m}$ wavelength regime. Several star forming complexes were observed, including NGC 6334, Chamaeleon I, $\eta$ Chamaeleontis, the Carina Nebula, 30 Doradus, RCW $57, \mathrm{RCW} 38$, as well as the Galactic Center. Images were obtained of lines at $2.42 \mu \mathrm{m} \mathrm{H}_{2}, 3.29 \mu \mathrm{m} \mathrm{PAH}$ and $4.05 \mu \mathrm{m} \mathrm{Br} \alpha$, as well as $3.5 \mu \mathrm{m} \mathrm{L-band} \mathrm{and}$ $4.7 \mu \mathrm{m} \mathrm{M}$-band continuum emission. These data, combined with near-IR, midIR, and radio continuum maps, reveal the environments of these star forming sites, as well as any protostars lying within them. The SPIREX project, its observing and reduction methods, and some sample data are summarized here.
\end{abstract}

\section{Introduction}

The South Pole Infra-Red EXplorer (SPIREX) telescope was a prototype system, developed to test the feasibility of building, operating, and maintaining an infrared (IR) telescope during an Antarctic winter. The initial driver for the SPIREX project was to exploit the conditions at the South Pole that make it an excellent site for 3-5 $\mu \mathrm{m}$ observations - that is the high altitude, the low temperatures, low precipitable water vapor content in the atmosphere, and the stable weather conditions (Burton et al. 1994; Marks et al. 1996; Hidas et al. 2000; Marks 2002).

The SPIREX $60 \mathrm{~cm}$ telescope began operations at the Amundsen-Scott South Pole Station in 1994 (Hereld et al. 1990). SPIREX was initially used as part of a campaign to measure the South Pole's thermal background, sky transparency, and the fraction of time useful for IR observations (see e.g. Ashley et al. 1996, Nguyen et al. 1996, Phillips et al. 1996 and Chamberlain et al. 2000 for further details on the IR sky conditions).

Due to the very low thermal background, SPIREX could perform longer integrations in the 3-5 $\mu \mathrm{m}$ regime, compared to temperate latitude facilities. In addition to continuum emission which, at these wavelengths pinpoints young embedded objects, there are many astrophysically significant molecular lines 
Table 1. Parameters of the filters and achieved sensitivities for SPIREX/Abu.

\begin{tabular}{|l|ccc|c|c|}
\hline Filter & $\begin{array}{c}\text { Center } \\
\mu \mathrm{m}\end{array}$ & $\begin{array}{c}\text { Width } \\
\mu \mathrm{m}\end{array}$ & $\begin{array}{c}\text { Range } \\
\mu \mathrm{m}\end{array}$ & ${\text { Time } \times \text { Coadds }^{b}}$ & $\begin{array}{c}\text { Sensitivity }^{c} \\
3 \sigma, 5^{\prime \prime} \times 5^{\prime \prime}, 1 \text { hour }\end{array}$ \\
\hline $\mathrm{H}_{2}{ }^{a}$ & 2.425 & 0.034 & $2.408-2.441$ & $360 \times 1$ & $6 \times 10^{-18} \mathrm{Wm}^{-2}$ \\
$\mathrm{PAH}$ & 3.299 & 0.074 & $3.262-3.336$ & $60 \times 3$ & $1 \mathrm{mJy}^{-2}$ \\
$\mathrm{Br} \alpha$ & 4.051 & 0.054 & $4.024-4.078$ & $10 \times 18$ & $5 \times 10^{-17} \mathrm{Wm}^{-2}$ \\
\hline $\mathrm{L}$ & 3.514 & 0.618 & $3.205-3.823$ & $6 \times 30$ & 14.6 mags \\
$\mathrm{L}$ & 3.821 & 0.602 & $3.520-4.122$ & $6 \times 30$ & 14.6 mags \\
narrow M & 4.668 & 0.162 & $4.586-4.749$ & $1.2 \times 90$ & 10.2 mags \\
\hline
\end{tabular}

${ }^{a}$ Covering the (1-0) $\mathrm{Q}(1)-\mathrm{Q}(5)$ lines.

${ }^{b}$ Typical integration time (in seconds) $\times$ number of coadded frames per position.

${ }^{c}$ Achieved sensitivities were up to 1 magnitude worse than theoretical sensitivities for the site with optimal instrument performance.

accessible; e.g. those from hydrogen and PAH molecules. Observations at 3$5 \mu \mathrm{m}$ are difficult at most temperate sites, making studies involving these lines limited, and thus the SPIREX dataset unique.

From 1994-1997 SPIREX was equipped with the GRIM (grism imager) camera. This detector contained $128 \times 128$ pixels and was sensitive from 1$2.5 \mu \mathrm{m}$. In 1998 the telescope was equipped and with the Abu camera, which incorporated an engineering grade $1024 \times 1024$ Aladdin detector (Fowler et al. 1998). This camera was sensitive from $2.4-5 \mu \mathrm{m}$, and provided a $10^{\prime}$ field of view image with a $0.6^{\prime \prime}$ pixel scale. Six science filters were available with SPIREX/Abu. The three narrow-band filters were optimized to isolate emission from molecular hydrogen $\left(\mathrm{H}_{2}\right.$ at $\left.2.42 \mu \mathrm{m}\right)$, polycyclic aromatic hydrocarbons (PAHs at $3.29 \mu \mathrm{m}$ ), and hydrogen line emission ( $\mathrm{Br} \alpha$ at $4.05 \mu \mathrm{m})$. The three broad-band filters covered the $\mathrm{L}^{-}, \mathrm{L}$ '- and $\mathrm{M}$-bands. The filter parameters and achieved sensitivities are listed in Table 1 (see Burton et al., 2000).

SPIREX/Abu was well suited for studies of star forming complexes. Young stars containing circumstellar disks have a color excess in the IR due to the absorption and re-emission of radiation from the central star by the surrounding material. Recent studies have found that the L-band may be the optimal wavelength for detection of star and disk systems. Compared to the $(\mathrm{H}-\mathrm{K})$ color $[\equiv 1.6-2.2 \mu \mathrm{m}]$, the $(\mathrm{K}-\mathrm{L})$ color $[\equiv 2.2-3.5 \mu \mathrm{m}]$ is more sensitive to the presence of a disk (Haisch et al. 2000; Lada et al. 2000; Kenyon \& Hartmann 1995).

Observations of PAH molecular line emission across star forming complexes, allows one to study their environments. The fluorescent emission from $\mathrm{PAH}$ molecules trace regions (known as photo dissociation regions or PDRs), where stellar UV radiation is heating the molecular gas (Hollenbach \& Tielens 1997). PAH emission delineates externally heated molecular clouds and reveals the interactions between nearby massive stars and any remnant molecular material.

The first astronomical results from SPIREX were obtained when ShoemakerLevy 9 collided with Jupiter in 1994. Using the GRIM camera at a wavelength of $2.36 \mu \mathrm{m}$, images were obtained at 5 minute intervals and captured 16 of the fragments, showing evidence of impact with Jupiter in 10 cases (Severson 2000). An extended halo around the edge-on spiral galaxy ESO 240-G11 was also im- 
aged using GRIM at $2.4 \mu \mathrm{m}$ (Rauscher et al., 1998), reaching a sensitivity level of $25 \mathrm{mags} / \operatorname{arcsec}^{2}$.

The following sections discuss a sample of data obtained from the two years of operation of SPIREX/Abu (1998 99) at the South Pole. Observations were conducted toward a number of different complexes. The characteristics of these range from young to old, low- to high-mass, and near and far star forming complexes. In particular, results are presented here for NGC 6334, Chamaeleon I, $\eta$ Chamaeleontis, the Carina Nebula, 30 Doradus, RCW 57, RCW 38, and the Galactic Center. In addition, SPIREX was used to search for an infrared counterpart to the gamma-ray burst GRB990705 at $3.5 \mu \mathrm{m}$ (Masetti et al., 2000), though no source was detected to a limit of 13.9 magnitudes after 2 hours of integration.

\section{Data Acquisition and Reduction}

For each source position, a series of frames were obtained at the specified integration time and then averaged. These parameters varied depending on the observing wavelength, the properties of the filter (narrow- or broad-band) and the weather conditions. Typical values for each filter are given in Table 1. All observations were conducted by the winter-over scientists at the South Pole station; Rodney Marks (1998) and Charlie Kaminsky (1999).

The sequence for all observations consisted of a set of sky frames followed by two sets of object frames. Each set consisted of five averaged frames offset by $\sim 30^{\prime \prime}$ from the previous. This sequence was repeated allowing the easy removal of sky emission and artifacts from the array. Archived images were used for dark subtraction and flat-fielding. Observations of standard stars were obtained before and after all on-source observations for flux calibration.

The majority of the data presented here was reduced by Joel Kastner using the SPIREX/Abu data pipeline ${ }^{12}$ (the exceptions are NGC 6334 and the Carina Nebula). The data pipeline was a joint project of the Rochester Institute for Technology Center for Imaging Science (RIT CIS), the National Optical Astronomy Observatories (NOAO) and the Center for Astrophysical Research in Antarctica (CARA).

To cover the star forming complexes of NGC 6334 and the Carina Nebula, many adjacent positions were observed. Common stars in adjacent frames were used to align the images and create the final larger mosaic (using IRAF routines written by Peter McGregor ${ }^{13}$ ). The final PAH-band mosaiced image for the Carina Nebula contained a total of 72 individual images, and for NGC 6334 the mosaic PAH-band image contained 304 images.

\footnotetext{
${ }^{12}$ See http://pipe.cis.rit.edu/

${ }^{13}$ See http://www.mso.anu.edu/observing/2.3m/CASPIR/
} 


\section{Results and Discussion}

\subsection{NGC 6334}

NGC 6334 is a young massive star forming region at a distance of $1.7 \mathrm{kpc}$. It contains seven distinct sites of ongoing star formation along a central molecular ridge. Fig. 1(a) shows the 3.29- $\mu \mathrm{m}$ data toward NGC 6334.

The emission within this band comprises both PAH and continuum emission. A massive embedded star is seen at each of several sites of star formation along the ridge, each $\sim 1 \mathrm{pc}$ apart. They are surrounded by complex loops and filaments of PAH emission. These PDRs have formed on the edge of the remnant molecular cloud, as radiation from the young stars carves the material and ionizes their surroundings. Analysis of the PAH emission and PDR features across the central ridge of NGC 6334 have been presented in Jackson et al. (1999) and Burton et al. (2000). The data shown here expand on these studies, and further reveal the PDR structure adjacent to the central star forming ridge.

In addition to the $\mathrm{PAH}-$ band images shown here, $\mathrm{L}-$ band images were also obtained across NGC 6334 . When combined with near-IR data from the 2MASS point source catalog they allow us to produce near-IR color-color diagrams (e.g., $(\mathrm{J}-\mathrm{H})$ vs. $(\mathrm{H}-\mathrm{K})$ and $(\mathrm{J}-\mathrm{H})$ vs. $(\mathrm{K}-\mathrm{L})$ diagrams). Results presented in Rathborne et al. (2003) find 11 sources with a large IR excess using the (K-L) color, compared to just a single source using the $(\mathrm{H}-\mathrm{K})$ color excess, confirming that the $(\mathrm{K}-\mathrm{L})$ color is far more sensitive to the detection of circumstellar disks.

\subsection{Chamaeleon I}

Containing in excess of 100 pre-main sequence stars, the Chamaeleon I dark cloud is one of the most active regions of nearby low-mass star formation. The central $0.5 \mathrm{deg}^{2}$ of the complex was observed in the L-band using SPIREX/Abu. These images reveal all of the known pre-main sequence stars (to an $L \leq 11$ ).

Kenyon \& Gómez (2001) combined JHK observations obtained at the CTIO with the SPIREX/Abu data, to construct near-IR colour-colour diagrams. They find the fraction of sources with an IR excess to be $58 \pm 4 \%$ (complete to an L $<11$ ). In addition, they also confirm that sources with an IR excess are more easily identified when using the $(\mathrm{K}-\mathrm{L})$ colour than $(\mathrm{H}-\mathrm{K})$.

\section{3. $\quad \eta$ Chamaeleontis}

The $\eta$ Chamaeleontis cluster is one of the nearest to the Sun, lying just $97 \mathrm{pc}$ away. It is also of intermediate age for a pre-main sequence system, $\sim 9$ Myrs old, and somewhat older than a number of other clusters where the formation and evolution of circumstellar disks has been studied. Importantly, its proximity and compactness mean that there is a complete population census for its members - 15 stars ranging from 0.2 to $3.4 \mathrm{M}_{\odot}$ in mass. Lyo et al. (2003) imaged the cluster using SPIREX/Abu in the L-band, finding $60 \%$ of the stars had IR excesses attributable to the presence of disks around them. Of those with disks, half showed a clear relation between the strength of the IR excess at $3.5 \mu \mathrm{m}$ and the equivalent width of the $\mathrm{H} \alpha$ line emission, implying continuous accretion. The lifetime of the disks in $\eta$ Chamaeleontis ( $\sim 9 \mathrm{Myrs})$ is significantly longer than in a number of other systems that have been studied, with lifetimes of 3-6 Myrs (e.g., Haisch et al., 2001). 

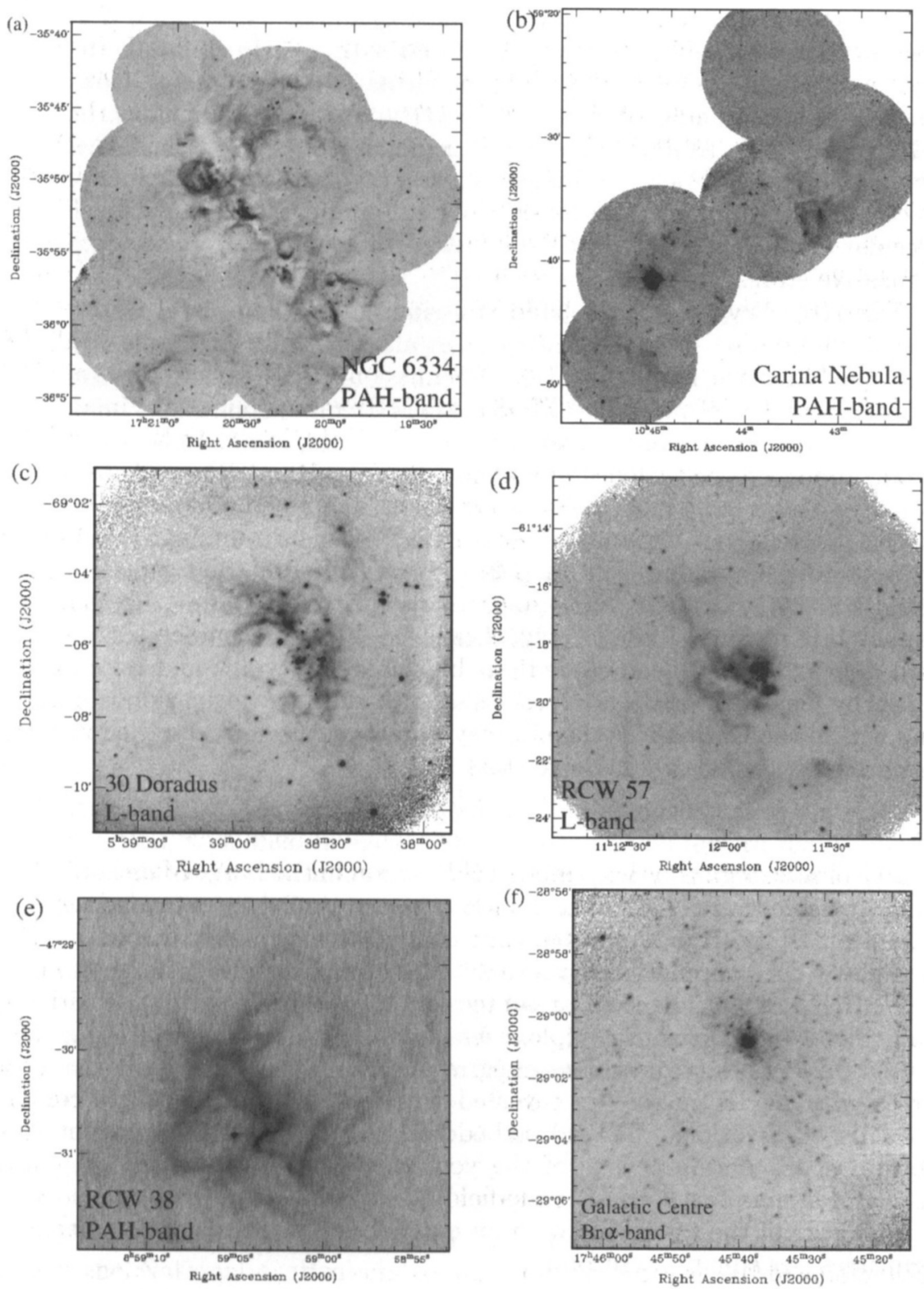

Figure 1. A collection of the images obtained with SPIREX/Abu. (a) NGC 6334 in $3.29 \mu \mathrm{m}$ PAH emission (Burton et al., 2000, Rathborne et al., 2004), (b) The Carina Nebula in PAH emission (Rathborne et al., 2002), (c) 30 Doradus in $3.5 \mu \mathrm{m}$ L-band emission, (d) RCW 57 in L-band, (e) RCW 38 in PAH emission and (f) the Galactic Center in the $4.05 \mu \mathrm{m}$ Br $\alpha$ filter. The RCW 38 and 57 images were kindly provided by Chris Wright. 


\subsection{The Carina Nebula}

The Carina Nebula is a massive star forming region at a distance of $2.2 \mathrm{kpc}$. Because no protostellar objects had been found within this nebula, it was thought to be 'evolved' and devoid of current star formation activity (e.g., Cox \& Bronfman 1995). It does however, contain 53 O-type stars and includes the clusters $\operatorname{Tr} 14$ and $\operatorname{Tr} 16$. At its centre lies the massive star $\eta$ Car and the Keyhole Nebula. Optical and near-IR images show a high amount of extinction toward this complex, with many dark lanes, globules, clumps, and filaments. Two large molecular clouds are located at the edges of the complex, in close proximity to the massive stars.

Fig. 1(b) shows the PAH-band emission across the central Carina Nebula (these data are also discussed in Brooks et al. 2000 and Rathborne et al. 2002). When combined with $2.12-\mu \mathrm{m} \mathrm{H}_{2}$ line emission, MSX 8- and $21-\mu \mathrm{m}$ images, SEST molecular line maps, and MOST $843 \mathrm{MHz}$ radio continuum images, three different environments are revealed across the complex: (i) the Keyhole nebula containing discreet, dense molecular clumps with PDRs on their surfaces; (ii) PDRs at the edges of both the southern and northern molecular clouds; and (iii) heated dust, intermixed with the PDRs surrounding $\operatorname{Tr} 14$ and the northern molecular cloud. Several 3.29-, $8^{-}$and $21-\mu \mathrm{m}$ point sources were also located across the complex, with spectral energy distributions corresponding to compact HII regions. Interestingly, these were all found on the edges of PDRs which appear to have been carved out by the stellar winds and radiation from the nearby massive stars. These results suggest that star formation is indeed ongoing within the Carina Nebula and may in fact be triggered by the interactions resulting from the nearby massive stars.

\subsection{Doradus}

The 30 Doradus region is the brightest $\mathrm{H}$ II region in the Large Magellanic Cloud. It contains a central cluster of massive stars surrounded by extended nebulosity in the near-IR, with many protostellar candidates. To study the nature of the embedded stellar population in more detail, L-band observations were obtained with SPIREX/Abu. The data presented in Fig. 1(c) are the most sensitive ever obtained of the 30 Doradus complex. The faintest start seen in the image, which required 9.25 hours of on-source integration, has $\mathrm{L}=14.5$ magnitudes. Many sources with an IR excess are revealed when the L-band data are combined with JHK observations. Several embedded massive stars are apparent and in addition, a population census of the young stellar objects can be conducted. This still remains the deepest, wide-field L-band image ever obtained, despite the small size of the telescope, with an extended source sensitivity $(1 \sigma)$ of 18.2 magnitudes per square arcsecond.

\subsection{RCW 57}

RCW 57 is a bright southern H II region. It contains a tight cluster of massive stars and shows extended infrared nebulosity. L-band observations of the central $10^{\prime}$ of this complex were obtained with SPIREX/Abu (Fig. 1(d)). This image reveals many point sources, in addition to both bright and diffuse extended emission. Using JHK and the SPIREX/Abu L-band data, studies are currently underway into the star formation history of this complex. 


\section{7. $\quad$ RCW 38}

RCW 38 is also a bright H II region containing a tightly packed cluster of stars. Near-IR images of this complex show extended nebulosity, with dust lanes and dark patches. Coincident with many of these features is extended $3.29-\mu \mathrm{m} \mathrm{PAH}$ emission, as shown in Fig. 1(e). These features trace the PDRs and delineate the molecular material. In addition, they wrap around the ionized material seen at $2 \mu \mathrm{m}$.

\subsection{The Galactic Centre}

Observations of the Galactic Centre obtained with SPIREX/Abu are far less affected by the extinction than at optical and near-IR wavelengths. The $4.05 \mu \mathrm{m}$ image in Fig. 1(f) clearly demonstrates the advantages of this wavelength regime. Most of the $4.05 \mu \mathrm{m}$ emission originates from heavily extincted sources, though there is also some $\operatorname{Br} \alpha$ emission, pinpointing regions of ionized gas. Three sources, of roughly equal brightness, are prominent in the nucleus at $4 \mu \mathrm{m}$. This is in contrast to the view at $2 \mu \mathrm{m}$, which is dominated by the source IRS 7 . These three sources are each separated by $\sim 7^{\prime \prime}$ and centred roughly on the presumed nucleus, Sgr A*. At the spatial resolution achieved with SPIREX they are associated with the sources IRS 1W, IRS 7/IRS 3 and IRS 13E/IRS 2L, respectively (as imaged by Clenet et al. 2001, in the L-band).

\section{Conclusions}

Although SPIREX/Abu was a prototype facility, it nevertheless obtained deep, high-resolution, wide-field images in the $3-5 \mu \mathrm{m}$ regime toward many star forming complexes. These data are not only unique, but when combined with complementary near-, mid-IR, and radio continuum observations, reveal the inner environments of star forming complexes (by tracing the PDRs), and identify the youngest objects with circumstellar disks (through their high L-band fluxes). The success of the SPIREX/Abu system lends strong support to current plans to build larger IR telescopes on the Antarctic plateau, where they would provide the most sensitive facilities for $3-5 \mu \mathrm{m}$ observations on the Earth.

\section{Acknowledgments}

The SPIREX project was a collaboration between the USA and Australia, involving the National Optical Astronomy Observatories (NOAO), the United States Naval Observatory (USNO), the Center for Astrophysical Research in Antarctica (CARA), Boston University, Goddard Spaceflight Center (GSFC), Ohio State University (OSU), Rochester Institute of Technology (RIT), the University of Chicago (UC), the University of New South Wales (UNSW), the Australian National University (ANU) and the Universities Space Research Association (USRA). We are indebted to the dedicated efforts of our many colleagues here who together have demonstrated that infrared astronomy can indeed be conducted from the Antarctic plateau. 


\section{References}

Ashley, M. C. B. et al., 1996, PASP, 108, 721

Brooks, K. J. et al., 2000, MNRAS, 319, 95

Burton, M. G. et al., 1994, PASA, 11, 127

Burton, M. G. et al., 2000, ApJ, 542, 359

Cox, P. \& Bronfman, L., 1995, A\&A, 299, 583

Chamberlain, M. A. et al., 2000, ApJ, 535, 501

Clenet, Y. et al., 2001, A\&A, 376, 124

Fowler, A. et al., 1998, Proc. SPIE., 3354, 1170

Haisch, K. E., Lada E. A., Lada C. J., 2000, AJ, 120, 1396

Haisch, K. E., Lada, E. A., Lada, C. J., 2001, ApJL, 553, L153

Hereld, M. et al. 1990, Proc. SPIE, 1235, 43

Hidas, M. G. et al., 2000, PASA, 17, 260

Hollenbach D., Tielens A. G. G. M., 1997, ARA\&A, 35, 179

Jackson, J. M. et al., 1999, BAAS, 194, 7117

Kenyon, S. J., Gómez, M., 2001, AJ, 121, 2673

Kenyon, S. J., Hartmann L. W., 1995, ApJS, 101, 117

Lada, C. J. et al., 2000, ApJ, 120, 3162

Lyo, A.-R. et al., 2003, MNRAS, 338, 616

Marks, R. D., 2002, A\&A, 385, 328

Marks, R. D. et al., 1996, A\&AS, 118, 385

Masetti, N. et al., 2000, A\&A, 354, 473

Nguyen, H. et al., 1996, PASP, 108, 718

Phillips, A. et al., 1999, ApJ, 527, 1009

Rathborne, J. M. et al., 2004, A\&A, 418, 563

Rathborne, J. M. et al., 2002, MNRAS, 331, 85

Rauscher, B. J. et al., 1998, ApJ, 506, 116

Severson, S. 2000, PhD thesis, University of Chicago 